Research Paper

\title{
Female Specific Association between NNMT Gene and Schizophrenia in a Han Chinese population
}

\author{
Guo-xia Wang1,2*, Yong Zhang ${ }^{3 *}$,Zhuang-wei Lv ${ }^{4}$, Mao Sun ${ }^{1,2}$, Dan $W^{1,2}$, Xin-yu Chen ${ }^{1,2}$, Yuan-ming \\ $\mathrm{Wu}^{1,2}$ \\ 1. Center for DNA Typing, the Fourth Military Medical University, Xi'an, 710032, China \\ 2. Department of Biochemistry and Molecular Biology, the Fourth Military Medical University, Xi'an, 710032, China \\ 3. Department of Anatomy, Histology and Embryology, K. K. Leung Brain Research Centre, the Fourth Military Medical University, Xi'an, \\ 710032, China \\ 4. Department of Clinical Laboratory, the Third Affiliated Hospital, Xinxiang Medical University, Xinxiang, 453003, China
}

*These authors are equally contributed to this work and should be regarded as co-first authors.

$\square$ Corresponding author: Center for DNA Typing, Department of Biochemistry and Molecular Biology, The Fourth Military Medical University, No. 17Changle West Road, Xi'an, 710032, China, Tel.: +86 29 84774978;fax: +86 2983280696.E-mail: wuym@fmmu.edu.cn.

() Ivyspring International Publisher. This is an open-access article distributed under the terms of the Creative Commons License (http://creativecommons.org/ licenses/by-nc-nd/3.0/). Reproduction is permitted for personal, noncommercial use, provided that the article is in whole, unmodified, and properly cited.

Received: 2014.04.17; Accepted: 2014.08.27; Published: 2014.09.19

\begin{abstract}
Accumulating evidence has shown that alterations in one carbon metabolism might play an important role in the pathogenesis of schizophrenia (SZ). Nicotinamide-N-methyltransferase (NNMT) is one of the key enzymes of one-carbon metabolism. To examine whether NNMT gene was associated with SZ in Han Chinese population, we selected seven single nucleotide polymorphisms (SNPs) in NNMT gene, and investigated its association with SZ from a cohort of $42 \mathrm{SZ}$ patients and 86 healthy controls by Mass-ARRAY technology. Statistical analyses revealed that one ( $r$ 694539) of the SNPs in the female subgroup showed significant difference between SZ patients and controls both in genotypic $(p=0.0170)$ and allelic frequencies $(p=0.0059)$. We also found that the frequency of haplotype 'A G G C T C T' in the female patients was significantly higher than in controls $(p=0.0015)$. Our results suggest that NNMT rs694539 may have a role in the etiology of $\mathrm{SZ}$ in a Han Chinese female population.
\end{abstract}

Key words: Schizophrenia (SZ),nicotinamide-N-methyltransferase(NNMT), polymorphisms, rs694539.

\section{Introduction}

Schizophrenia (SZ) is a genetically complex and heterogeneous psychiatric disorder with a lifetime prevalence estimated at approximately $1 \%$ in worldwide populations [1]. The disorder is characterized by distortion of reality, delusions, hallucinations, altered emotional reactivity, disorganized behavior, social isolation and cognitive impairment [2]. There are gender differences in the onset and prevalence of SZ. Males with SZ have a 5-year younger age of onset and $40 \%$ greater risk than the females [3]. A combination of genetic and environmental factors plays a role in the development of SZ and the former one accounts for $80 \%$ to $90 \%$ of SZ [4]. Accumulated genetic asso- ciation studies have acknowledged that SZ is a polygenic disorder though the effect of each gene is small and the penetrance are still largely unknown [5, 6]. The exact etiology and molecular mechanism leading to the onset of SZ symptoms remains unclear. Mounting evidences suggest that alterations in one carbon metabolism might play an important role in the pathogenesis of SZ $[7,8]$.

Methylenetetrahydrofolate reductase (MTHFR) is one of the key enzymes of one-carbon metabolism. The C677T allele of MTHFR, associated with higher plasma homocysteine [9], was also found to be associated with neuropsychiatric disorders including SZ 
[10-12]. Another enzyme involved in one-carbon metabolism is Nicotinamide-N-methyltransferase (NNMT). Human NNMT (EC 2.1.1.1), a cytoplasmic enzyme belonging to Phase II conjugating enzymes, is expressed in brain and other nervous system [13]. This gene spans $16.5 \mathrm{~kb}$ on chromosome 11q23.1 and consists of three exons and two introns. NNMT catalyzes the transfer of methyl group from $S$-adenosyl-L-methionine (SAM) to nicotinamide (NA), generating 1-methylnicotinamide (1MNA) and $S$-adenosylhomocysteine (SAH) which is later hydrolyzed to homocysteine [14]. Homocysteine is one of the key components of one-carbon metabolism. Elevated plasma homocysteine levels were demonstrated to be associated with an increased risk of SZ [15-18], whereas neurocognitive improvement was obtained in patients with SZ after the reduction of homocysteine levels [19]. Two studies found that NNMT gene was significantly associated with hyperhomocysteinemia [20, 21]. Giusti et al. [22] found an association of polymorphisms across the NNMT gene with abdominal aortic aneurism and De Jonge et al. suggested an association between NNMT and paediatric acute lymphoblastic leukaemia. In an Israeli population, NNMT gene was shown to be associated with SZ [23]. Recently, Ali Sazci et al. showed that NNMT gene is a genetic risk factor for bipolar disorder and nonalcoholic steatohepatitis (NASH) in the Turkish population [24, 25].

Since no report regarding NNMT and SZ association in Han Chinese population, we conducted the first genetic association study to investigate the associations of the seven NNMT SNPs with SZ in a case-control study. Whether the sex-specific genetic associations of these polymorphisms exist in the Han population was also explored in this preliminary study.

\section{Material and methods}

\section{Subjects}

The case group comprised 21 females and 21 males aged from 15 to 53 yrs. (mean age $\pm S D=26.8 \pm$ 9.1 years old). The control group comprised 53 females and 33 males aged from 20 to 58 yrs (mean age $\pm \mathrm{SD}=31.2 \pm 9.0$ years old). All subjects were unrelated Chinese Han nationality. The patients were recruited from the Institute of Psychosomatic Clinic of the Third Affiliated Hospital of Xinxiang Medical University in Henan Province. They had been hospitalized for less than 1 month and fulfilled the Diagnostic and Statistical Manual of Mental Disorders, Fourth Edition (DSM-IV) criteria for SZ based on the diagnostic consensus of two experienced psychiatrists. The exclusion criteria were: other psychiatric disorders, neurological disorders, a history of significant drug abuse, drug use in the past year. The healthy controls were from the same geographical region as the patients and were screened to discard psychiatric disease using the 28-scaled global health questionnaire [26].

This study was approved by the Medical Ethics Committee of the Fourth Military Medical University and the Medical Ethics Committee of the School of Psychosomatic Clinic and the Center of Physical Examination of the Third Affiliated Hospital of Xinxiang Medical University. Signed informed consent documents were obtained from all participants.

\section{DNA extraction}

Peripheral whole blood from all 128 individuals was treated with EDTA-Na 2 for anticoagulationand stored at $-20^{\circ} \mathrm{C}$. Genomic DNA was extractedwith the RelaxGene Blood DNA System DP319-02 (TIANGEN, China). The concentration of genomic DNA was measured with NanoDrop 2000 (Thermo Scientific, USA).

\section{Genotyping}

Based on the HapMap database (http://www.hapmap.org), the NCBI SNP database (www.ncbi.nlm.gov/projects/SNP/), we selected 7 SNPs with minor allele frequencies (MAFs) $>5 \%$ according to the HapMap database for $\mathrm{CHB}$ (http://www.hapmap.org). SNP genotyping was performed using MassArray system (Sequenom, USA). The primer sequences are summarized in Supplementary Material: Table S1. The DNA samples were amplified on GeneAmp PCR System 9700 (Applied Biosystems, USA) and the PCR products were subjected for locus-specific single-base extension reactions. The resulting products were desalted and transferred to a 384 SpectroCHIP array. Allele detection was performed by a MALDI-TOF mass spectrometer. The mass spectrograms were analyzed by the Mass ARRAY Typer software version 3.4 (Sequenom, USA).

\section{Statistical analysis}

Each SNP was tested for violation of the Hardy-Weinberg Equilibrium (HWE) using Chi-square tests (see Table 1). We analyzed differences in the allelic and genotypic distribution frequencies between SZ patients and healthy controls for each SNP with Pearson's Chi-square tests (SPSS 13.0 software Chicago, IL, USA). Haplotype frequencies were assessed by the SHEsis software [27]. Moreover, Gender specific effect were considered and analyzed. Linkage disequilibrium (LD) analysis using the free online software Haploview version 4.1 (http://www.broad. mit.edu/mpg/haploview/) were performed to ex- 
amine haplotype impact on SZ.P-values below 0.05 were considered statistically significant.

\section{Results}

\section{Comparison of the allelic and genotypic frequencies of SNPs in patients and control group}

We chose 7 SNPs (rs694539, rs2256292, rs2301128, rs10891645, rs2155806, rs1941398, rs2604279) in the NNMT gene for genotyping. The position and the location of these 7 SNPs are shown in Table 1. No significant deviation from the HWE was found in either the patient or control group for all seven SNPs $(p>0.05)$. Statistical analysis revealed no significant differences in the frequencies of the genotype and allele between the patients and controls (Supplementary Material: Table S2).
To determine the gender effect, genotype and allele frequencies in both sexes were assessed (Table 2). In female samples, the rs694539 showed significant difference between SZ patients and controls in the genotype $(p=0.0170)$ and allele $(p=0.0059, \mathrm{OR}=3.009$, $95 \% \mathrm{CI}=1.348 \sim 6.716)$, which was not found in males.

Table I. The position and the location of these seven SNPs.

\begin{tabular}{lllll}
\hline SNP & Alleles & Location & $\begin{array}{l}\text { Chromosome } \\
\text { Position(chr.11) }\end{array}$ & HWE(p) \\
\hline rs694539 & A/G & 5'near gene & 113638629 & 0.05165 \\
rs2256292 & C/G & Intron & 114167939 & 0.55661 \\
rs2301128 & A/G & Intron & 114167999 & 0.69132 \\
rs10891645 & A/C & Intron & 114171247 & 0.48133 \\
rs2155806 & $\mathrm{C} / \mathrm{G}$ & Intron & 114172510 & 0.69761 \\
rs1941398 & $\mathrm{C} / \mathrm{G}$ & intron & 114178056 & 0.71195 \\
rs2604279 & $\mathrm{C} / \mathrm{T}$ & Intron & 114178541 & 0.63002 \\
\hline
\end{tabular}

HWE: Hardy-Weinberg Equilibrium.

Table 2. Distribution of NNMT genotypes and alleles between SZ cases and controls in female and male samples.

\begin{tabular}{|c|c|c|c|c|c|c|c|c|c|}
\hline SNP & $\begin{array}{l}\text { Haplo- } \\
\text { type/Allele }\end{array}$ & Female Cases & Controls & $P$-value & $\mathrm{OR}(95 \% \mathrm{CI})$ & Male Cases & Controls & $P$-value & $\mathrm{OR}(95 \% \mathrm{CI})$ \\
\hline \multirow[t]{5}{*}{ rs694539* } & $\mathrm{AA}$ & $2(0.095)$ & $1(0.019)$ & & & $0(0.000)$ & $2(0.061)$ & & \\
\hline & AG & $12(0.571)$ & $16(0.302)$ & & & $9(0.429)$ & $17(0.515)$ & & \\
\hline & GG & $7(0.333)$ & $36(0.679)$ & 0.0170 & & $12(0.571)$ & $14(0.424)$ & 0.3588 & \\
\hline & A & $16(0.381)$ & $18(0.170)$ & & & $9(0.214)$ & $21(0.318)$ & & \\
\hline & G & $26(0.619)$ & $88(0.830)$ & 0.0059 & $3.009(1.348 \sim 6.716)$ & $33(0.786)$ & $45(0.682)$ & 0.2399 & $0.584(0.237 \sim 1.439)$ \\
\hline \multirow[t]{5}{*}{ rs2256292 } & $\mathrm{CC}$ & $1(0.048)$ & $9(0.170)$ & & & $4(0.190)$ & $1(0.030)$ & & \\
\hline & CG & $11(0.524)$ & $26(0.491)$ & & & $7(0.333)$ & $18(0.545)$ & & \\
\hline & GG & $9(0.429)$ & $18(0.340)$ & 0.3640 & & $10(0.476)$ & $14(0.424)$ & 0.0871 & \\
\hline & $\mathrm{C}$ & $13(0.310)$ & $44(0.415)$ & & & $15(0.357)$ & $20(0.303)$ & & \\
\hline & G & $29(0.690)$ & $62(0.585)$ & 0.2341 & $0.632(0.295 \sim 1.350)$ & $27(0.643)$ & $46(0.697)$ & 0.5581 & $1.278(0.562 \sim 2.904)$ \\
\hline \multirow[t]{5}{*}{ rs2301128 } & AA & $0(0.000)$ & $1(0.019)$ & & & & & & \\
\hline & AG & $4(0.190)$ & $11(0.208)$ & & & $3(0.143)$ & $7(0.212)$ & & \\
\hline & GG & $17(0.810)$ & $41(0.774)$ & 0.8015 & & $18(0.857)$ & $26(0.788)$ & 0.5229 & \\
\hline & $\mathrm{A}$ & $4(0.095)$ & $13(0.123)$ & & & $3(0.071)$ & $7(0.106)$ & & \\
\hline & G & $38(0.905)$ & $93(0.877)$ & 0.6374 & $0.753(0.231 \sim 2.457)$ & $39(0.929)$ & $59(0.894)$ & 0.5449 & $0.648(0.158 \sim 2.660)$ \\
\hline \multirow[t]{4}{*}{ rs10891645 } & $\mathrm{AC}$ & $2(0.095)$ & $5(0.094)$ & & & $3(0.143)$ & $5(0.152)$ & & \\
\hline & $\mathrm{CC}$ & $19(0.905)$ & $48(0.906)$ & 0.9905 & & $18(0.857)$ & $28(0.848)$ & 0.9304 & \\
\hline & A & $2(0.048)$ & $5(0.047)$ & & & $3(0.071)$ & $5(0.076)$ & & \\
\hline & $\mathrm{C}$ & $40(0.952)$ & $101(0.95)$ & 0.9907 & $1.010(0.188 \sim 5.421)$ & $39(0.929)$ & $61(0.924)$ & 0.9332 & $0.938(0.212 \sim 4.150)$ \\
\hline \multirow[t]{5}{*}{ rs2155806 } & $\mathrm{CC}$ & $1(0.048)$ & $0(0.000)$ & & & $1(0.048)$ & $0(0.000)$ & & \\
\hline & $\mathrm{CT}$ & $5(0.238)$ & $14(0.264)$ & & & $4(0.190)$ & $9(0.273)$ & & \\
\hline & $\mathrm{TT}$ & $15(0.714)$ & $39(0.736)$ & 0.2762 & & $16(0.762)$ & $24(0.727)$ & 0.3767 & \\
\hline & $\mathrm{C}$ & $7(0.167)$ & $14(0.132)$ & & & $6(0.143)$ & $9(0.136)$ & & \\
\hline & $\mathrm{T}$ & $35(0.833)$ & $92(0.868)$ & 0.5867 & $1.314(0.490 \sim 3.527)$ & $36(0.857)$ & $57(0.864)$ & 0.9242 & $1.056(0.346 \sim 3.216)$ \\
\hline \multirow[t]{5}{*}{ rs1941398 } & $\mathrm{CC}$ & $13(0.619)$ & $36(0.679)$ & & & $11(0.524)$ & $19(0.576)$ & & \\
\hline & CG & $7(0.333)$ & $17(0.321)$ & & & $7(0.333)$ & $13(0.394)$ & & \\
\hline & GG & $1(0.048)$ & $0(0.000)$ & 0.2707 & & $3(0.143)$ & $1(0.030)$ & 0.3036 & \\
\hline & $\mathrm{C}$ & $33(0.786)$ & $89(0.840)$ & & & $29(0.690)$ & $51(0.773)$ & & \\
\hline & G & $9(0.214)$ & $17(0.160)$ & 0.4372 & $0.700(0.284 \sim 1.725)$ & $13(0.310)$ & $15(0.227)$ & 0.3417 & $0.656(0.274 \sim 1.568)$ \\
\hline \multirow[t]{5}{*}{ rs2604279 } & $\mathrm{CC}$ & & & & & $2(0.095)$ & $0(0.000)$ & & \\
\hline & $\mathrm{CT}$ & $6(0.286)$ & $11(0.208)$ & & & $6(0.286)$ & $10(0.303)$ & & \\
\hline & $\mathrm{TT}$ & $15(0.714)$ & $42(0.792)$ & 0.4711 & & $13(0.619)$ & $23(0.697)$ & 0.1947 & \\
\hline & $\mathrm{C}$ & $6(0.143)$ & $11(0.104)$ & & & $10(0.238)$ & $10(0.152)$ & & \\
\hline & $\mathrm{T}$ & $36(0.857)$ & $95(0.896)$ & 0.5014 & $1.439(0.496 \sim 4.180)$ & $32(0.762)$ & $56(0.848)$ & 0.2588 & $1.750(0.658 \sim 4.655)$ \\
\hline
\end{tabular}


Table 3. Estimation of LD between each pair of Loci. in NNMT.

\begin{tabular}{|c|c|c|c|c|c|c|c|}
\hline & rs694539 & rs2256292 & rs2301128 & rs10891645 & rs2155806 & rs1941398 & rs2604279 \\
\hline rs694539 & - & 0.056 & 0.007 & 0.019 & 0.017 & 0.011 & 0.016 \\
\hline rs2256292 & 0.547 & - & 0.210 & 0.035 & 0.021 & 0.093 & 0.062 \\
\hline rs2301128 & 0.422 & 0.998 & - & 0.007 & 0.017 & 0.006 & 0.002 \\
\hline rs10891645 & 0.966 & 0.998 & 0.995 & - & 0.261 & 0.147 & 0.201 \\
\hline rs2155806 & 0.553 & 0.479 & 0.926 & 0.828 & - & 0.437 & 0.127 \\
\hline rs1941398 & 0.351 & 0.786 & 0.424 & 0.795 & 0.845 & - & 0.632 \\
\hline rs2604279 & 0.526 & 0.807 & 0.294 & 0.738 & 0.361 & 1.00 & - \\
\hline
\end{tabular}

$\mathrm{D}^{\prime}$-value is shown below the subtraction sign, and $\mathrm{r}^{2}$-value is shown above the subtraction sign.

Table 4. Comparison of haplotype frequencies for seven SNPs (rs694539, rs2256292, rs230II28, rs 10891645, rs2 I55806, rs I94I398, rs2604279) between SZ cases and controls.

\begin{tabular}{|c|c|c|c|c|c|}
\hline Haplotype & Case(freq) & Control(freq) & $\mathrm{Chi}^{2}$ & $P$-value & Odds Ratio $(95 \% \mathrm{CI})$ \\
\hline \multicolumn{6}{|l|}{ Total } \\
\hline A G GCTCT & $17.78(0.212)$ & 28.02(0.163) & 0.559 & 0.454821 & $1.290[0.661 \sim 2.521]$ \\
\hline GCACTCT & $7.00(0.083)$ & $15.60(0.091)$ & 0.111 & 0.738690 & $0.853[0.334 \sim 2.176]$ \\
\hline GCGCTCT & $17.19(0.205)$ & $38.66(0.225)$ & 0.369 & 0.543393 & $0.818[0.428 \sim 1.564]$ \\
\hline G GG A C G C & $4.95(0.059)$ & $6.98(0.041)$ & 0.305 & 0.580956 & $1.395[0.426 \sim 4.563]$ \\
\hline G GG C C G T & $3.79(0.045)$ & $4.13(0.024)$ & 0.688 & 0.406927 & 1.811 [0.437 7.507] \\
\hline G GG C T C T & $18.23(0.217)$ & $45.65(0.265)$ & 1.235 & 0.266395 & $0.701[0.373 \sim 1.314]$ \\
\hline G GG C T GC & $5.82(0.069)$ & $11.37(0.066)$ & 0.001 & 0.980412 & 0.987 [0.349 2.789] \\
\hline A G G C T GC & $3.18(0.038)$ & $0.00(0.000)$ & 6.222 & 0.012635 & \\
\hline \multicolumn{6}{|l|}{ Female } \\
\hline A G G C T C T* & $15.99(0.381)$ & 13.86(0.131) & 10.131 & 0.001465 & 3.780 [1.620 8.820] \\
\hline GCACTCT & $4.00(0.095)$ & $8.97(0.085)$ & 0.005 & 0.942124 & $1.047[0.303 \sim 3.617]$ \\
\hline GCGCCGC & $2.00(0.048)$ & $0.80(0.008)$ & 2.335 & 0.126449 & $6.081[0.708 \sim 52.257]$ \\
\hline GCGCTCT & $6.00(0.143)$ & $26.51(0.250)$ & 2.691 & 0.100917 & 0.448 [0.169 1.188] \\
\hline G GG A C G C & $2.00(0.048)$ & $3.99(0.038)$ & 0.035 & 0.852335 & $1.179[0.207 \sim 6.711]$ \\
\hline G GG C C G T & $3.00(0.071)$ & $3.00(0.071)$ & 0.253 & 0.615024 & $1.461[0.331 \sim 6.453]$ \\
\hline G GG C T C T & $7.00(0.167)$ & $32.25(0.304)$ & 3.857 & 0.050000 & $0.406[0.162 \sim 1.016]$ \\
\hline G GG C T G C & $1.00(0.024)$ & $4.49(0.042)$ & 0.168 & 0.681820 & $0.505[0.078 \sim 3.260]$ \\
\hline \multicolumn{6}{|l|}{ Male } \\
\hline A C A C TCT & $0.00(0.000)$ & $4.00(0.061)$ & 2.933 & 0.086768 & \\
\hline A C G C T C T & $1.70(0.040)$ & $4.56(0.069)$ & 0.553 & 0.457037 & $0.508[0.083 \sim 3.111]$ \\
\hline A G G C C G T & $0.01(0.000)$ & $2.00(0.030)$ & 0.775 & 0.378611 & $0.004[0.000 \sim 0.080]$ \\
\hline A G G C T C T & $3.63(0.086)$ & $8.44(0.128)$ & 0.713 & 0.398571 & $0.573[0.155 \sim 2.112]$ \\
\hline GCGCTCT & $9.64(0.229)$ & $5.72(0.087)$ & 3.492 & 0.061692 & $2.854[0.922 \sim 8.834]$ \\
\hline G GG C T C T & $12.05(0.287)$ & $26.24(0.398)$ & 2.537 & 0.111177 & 0.500 [0.212 1.179] \\
\hline G GG C T G C & $5.98(0.142)$ & $5.00(0.076)$ & 0.894 & 0.344342 & $1.830[0.516 \sim 6.484]$ \\
\hline A G G A C GC & $2.00(0.048)$ & $0.00(0.000)$ & 2.907 & 0.088176 & \\
\hline G GG C C G T & $2.33(0.055)$ & $0.00(0.000)$ & 3.398 & 0.065270 & \\
\hline
\end{tabular}

\section{LD relationships among the SNPs}

Table 3 presented the results of the LD tests (noted as $\mathrm{D}^{\prime}$ and $\mathrm{r}^{2}$ ) between each locus in NNMT. According to these results, $\mathrm{LD}\left(\mathrm{D}^{\prime}>0.8\right)$ was observed. As shown in Table 3, the associated SNP rs2256292 and two SNPs (rs10891645, rs2301128) were observed in the LD block for NNMT. The haplotype analysis did not reveal any significant association among SZ and the controls, which was also not found in males and females (Supplementary Material: Table S3). Haplotypes with seven loci (rs694539, rs2256292, rs2301128, rs10891645, rs2155806, rs1941398, rs2604279) of NNMT gene polymorphisms were analyzed (Table 4). All those frequency less than 0.03 will be ignored in analysis. We found no significant difference for haplotype in all cases and controls. When analyzed in the gender-specific samples, the frequency of haplotype 'A G G C T C T' was $38.1 \%$ of cases, which is significantly higher than in controls $(13.1 \%)$, and the odds ratios were $3.780(95 \%$ $\mathrm{CI}=1.620 \sim 8.820, p=0.0015)$, between the female patients and controls.

\section{Discussion}

$\mathrm{SZ}$ is a multifactorial disease, with contributions from multiple susceptibility genes, epigenetic, and environmental factors. Although the exact mechanism of SZ remains unknown, the role of impaired one carbon metabolism in the pathogenesis of psychiatric disorders has been studied by many researchers. Currently, we attempted to establish an association between the polymorphisms in NNMT genes and SZ 
in a Han Chinese population.

For the female subgroup, the rs694539, the frequencies of genotype and allele were significantly different between the SZ patients and the controls. We also found that the frequency of haplotype 'A G G C T $\mathrm{C} \mathrm{T}^{\prime}$ was significantly higher in the female patients than controls. Thus, the haplotype 'A G G C T C T' may have a higher risk for the female SZ. In previous research, the frequency of the SNP rs694539 was also reported to be significantly different between cases and control groups in many neurodegenerative diseases. The rs694539 variant has previously been found to be associated with elevated plasma homocysteine levels in 398 Spanish subjects [22]. Bromberg et al. found the rs694539 was associated with SZ in an Israeli population [26]. Ali Sazci et al. [24, 25] suggested that the rs694539 variant of NNMT gene was a genetic risk factor for bipolar patients and for developing NASH, and demonstrated a sex-dependent susceptibility within the NNMT gene for bipolar in a Turkish population. Interestingly, our study also identified a gender-related statistically significant association for the rs694539 in the genotypic and allelic comparison between the female SZ and controls, suggesting that the rs694539 variant of NNMT gene might play a role in female SZ.

The rs694539 SNP, located upstream (at the 114133419th bp, G>A transition; dbSNP) of the NNMT gene in the noncoding region, affects the regulation of transcription [23]. This impact is proposed to alter the cellular pathways, thus causing genetic risk for psychiatric disorders [28]. Therefore, the rs694539 was a key risk factor related to SZ. The role of this variant of NNMT gene in SZ disorder is unclear, though dysregulation of epigenetics and/or elevated homocysteine and/or disturbed the nicotinamide levels may be one of the underlying causes [27].

This study suffers from some limitations. The cohort being analyzed consists of a relatively small sample size. Etiological heterogeneity and clinical heterogeneity cannot be rule out as these may influence both the genotypic and allelic distribution of the NNMT gene in SZ. Further investigations using larger sample sizes and family-based studies will add valuable insight into the understanding of genetic predisposition in SZ.

In conclusion, the rs694539 variant of NNMT gene may play a role in the etiology of SZ in a Han Chinese female population.

\section{Supplementary Material}

Tables S1-S3.

http://www.medsci.org/v11p1234s1.pdf

\section{Acknowledgments}

We sincerely thank the patients and healthy volunteers for their participation in this study. This work was supported by the National Natural Science Foundation of China (Grant No.81100816, and 30600268), and the Key Project Foundation (Grant No. 2013FWPT-06) of Shaanxi Science and Technology Commission.

\section{Conflict of interest}

The authors report no conflicts of interest.

\section{References}

[1] Saha S, Chant D, Welham J, McGrath J. A systematic review of the prevalence of schizophrenia. PLoS Med. 2005;2: e141.

[2] Andreasen NC, Carpenter WJ. Diagnosis and classification of schizophrenia. Schizophr Bull. 1993;19: 199-214.

[3] Mulle JG. Schizophrenia genetics: progress, at last. Current Opinion In Genetics \& Development. 2012;22: 238-44.

[4] Picchioni MM, Murray RM. Schizophrenia. BMJ. 2007;335: 91-5.

[5] Bergen SE, Petryshen TL. Genome-wide association studies of schizophrenia: does bigger lead to better results? Curr Opin Psychiatry. 2012;25: 76-82.

[6] Rangaswamy T, Greeshma M. Course and outcome of schizophrenia. Int Rev Psychiatry. 2012;24: 417-22.

[7] Frankenburg FR. The role of one-carbon metabolism in schizophrenia and depression. Harv Rev Psychiatry. 2007;15: 146-60.

[8] Krebs MO, Bellon A, Mainguy G, Jay TM, Frieling H. One-carbon metabolism and schizophrenia: current challenges and future directions. Trends In Molecular Medicine. 2009;15: 562-70.

[9] Frosst P, Blom HJ, Milos R et al. A candidate genetic risk factor for vascular disease: a common mutation in methylenetetrahydrofolate reductase. Nature Genetics. 1995;10: 111-3.

[10] Ezzaher A, Mouhamed DH, Mechri A et al. Hyperhomocysteinemia in Tunisian bipolar I patients. Psychiatry Clin Neurosci. 2011;65: 664-71.

[11] Kempisty B, Mostowska A, Gorska I et al. Association of $677 \mathrm{C}>\mathrm{T}$ polymorphism of methylenetetrahydrofolate reductase (MTHFR) gene with bipolar disorder and schizophrenia. Neuroscience Letters. 2006;400: 267-71.

[12] Peerbooms OL, van Os J, Drukker M et al. Meta-analysis of MTHFR gene variants in schizophrenia, bipolar disorder and unipolar depressive disorder: evidence for a common genetic vulnerability? Brain Behavior And Immunity. 2011;25: 1530-43.

[13] Sano A, Endo N, Takitani S. Fluorometric assay of rat tissue $\mathrm{N}$-methyltransferases with nicotinamide and four isomeric methylnicotinamides. Chem Pharm Bull (Tokyo). 1992;40: 153-6.

[14] Aksoy S, Szumlanski CL, Weinshilboum RM. Human liver nicotinamide $\mathrm{N}$-methyltransferase. cDNA cloning, expression, and biochemical characterization. Journal Of Biological Chemistry. 1994;269: 14835-40.

[15] Akanji AO, Ohaeri JU, Al-Shammri SA, Fatania HR. Associations of blood homocysteine concentrations in Arab schizophrenic patients. Clinical Biochemistry. 2007;40: 1026-31.

[16] Applebaum J, Shimon H, Sela BA, Belmaker RH, Levine J. Homocysteine levels in newly admitted schizophrenic patients. J Psychiatr Res. 2004;38: 413-6.

[17] Nishi A, Numata S, Tajima A et al. Meta-analyses of Blood Homocysteine Levels for Gender and Genetic Association Studies of the MTHFR C677T Polymorphism in Schizophrenia. Schizophr Bull. 2014.

[18] Regland B, Johansson BV, Grenfeldt B, Hjelmgren LT, Medhus M. Homocysteinemia is a common feature of schizophrenia. J Neural Transm Gen Sect. 1995;100: 165-9.

[19] Levine J, Sela BA, Osher Y, Belmaker RH. High homocysteine serum levels in young male schizophrenia and bipolar patients and in an animal model. Prog Neuropsychopharmacol Biol Psychiatry. 2005;29: 1181-91.

[20] Souto JC, Blanco-Vaca F, Soria JM et al. A genomewide exploration suggests a new candidate gene at chromosome $11 \mathrm{q} 23$ as the major determinant of plasma homocysteine levels: results from the GAIT project. American Journal Of Human Genetics. 2005;76: 925-33.

[21] Zhang L, Miyaki K, Araki J, Nakayama T, Muramatsu M. The relation between nicotinamide $\mathrm{N}$-methyltransferase gene polymorphism and plasma homocysteine concentration in healthy Japanese men. Thrombosis Research. 2007;121: 55-8.

[22] Giusti B, Saracini C, Bolli P et al. Genetic analysis of 56 polymorphisms in 17 genes involved in methionine metabolism in patients with abdominal aortic aneurysm. Journal Of Medical Genetics. 2008;45: 721-30.

[23] Bromberg A, Lerer E, Udawela $M$ et al. Nicotinamide-N-methyltransferase (NNMT) in schizophrenia: genetic association and decreased frontal cortex mRNA levels. Int J Neuropsychopharmacol. 2012;15: 727-37. 
[24] Sazci A, Ozel MD, Ergul E, Onder ME. Association of nicotinamide-N-methyltransferase (NNMT) gene rs694539 variant with bipolar disorder. Gene. 2013;532: 272-5.

[25] Sazci A, Ozel MD, Ergul E, Aygun C. Association of nicotinamide-N-methyltransferase gene rs694539 variant with patients with nonalcoholic steatohepatitis. Genet Test Mol Biomarkers. 2013;17: 849-53.

[26] Roig B, Virgos C, Franco $\mathrm{N}$ et al. The discoidin domain receptor 1 as a novel susceptibility gene for schizophrenia. Mol Psychiatry. 2007;12: 833-41.

[27] Shi YY, He L. SHEsis, a powerful software platform for analyses of linkage disequilibrium, haplotype construction, and genetic association at polymorphism loci. Cell Research. 2005;15: 97-8.

[28] Saito S, lida A, Sekine A et al. Identification of 197 genetic variations in six human methyltranferase genes in the Japanese population. I Hum Genet. 2001;46: 529-37. 